\title{
Stellar Oscillations in Giant Stars
}

\author{
A. P. Hatzes, ${ }^{1}$ M. P. Döllinger, ${ }^{2}$ M. Endl ${ }^{3}$ \\ 1 Thüringer Landessternwarte Tautenburg, Sternwarte 5, D-07778 Tautenburg, Germany \\ ${ }^{2}$ European Southern Observatory, Karl-Schwarzschild-Straße 2, D-85748 Garching, Germany \\ ${ }^{3}$ McDonald Observatory, The University of Texas at Austin, Austin, TX 78712, USA
}

\begin{abstract}
Walker et al. (1989) were the first to establish, using precise stellar radial velocity measurements, that $\mathrm{K}$ giant stars were a new class of variables. The variability of some of these stars can be quite complex showing several periods ranging from several hours to several hundreds of days. The long-period variations result from sub-stellar companions or rotational modulation, but the short-period variability certainly arises from stellar oscillations. We present recent of precise stellar radial velocity measurements $\left(\sigma \approx 6 \mathrm{~m} \mathrm{~s}^{-1}\right)$ of two oscillating $\mathrm{K}$ giant stars: $\beta$ Oph and $\gamma$ Dra. These stars show oscillation periods of hours $(\beta \mathrm{Oph})$ and days ( $\gamma$ Dra $)$. These periods are consistent with solar-like stellar oscillations, given the stellar properties of the star. Radial modes are a prime candidate for the type of oscillations in these stars.
\end{abstract}

\section{Introduction}

Over 15 years ago precise stellar radial velocity (RV) measurements established that $\mathrm{K}$ giant stars were a new class of variables (Walker et al. 1989). Subsequent investigations showed that these stars showed periodic variations on two time scales: hundreds of days (Hatzes \& Cochran 1993) and several days (Smith et al. 1987, Hatzes \& Cochran 1994a). Hatzes \& Cochran (1993) hypothesized that the long period variations could arise from either sub-stellar companions or rotational modulation. Later work revealed that planetary companions can indeed be the cause of some of the long period RV variations in these stars (Frink et al. 2000; Setiawan et al. 2003, 2005; Hatzes et al. 2005). More recently, Hatzes et al. (2006) showed that the long period variations in $\beta$ Gem originally found by Hatzes \& Cochran (1993) were indeed caused by a planetary companion.

Short-period RV variations ( $P=1.8$ days) were first found in $\alpha$ Boo by Smith et al. (1989). Hatzes \& Cochran (1994a) later found pulsation periods of 2.46 and 4.03 days (and possibly an 8.5 day period) in this star thus providing the first evidence for the presence of multiple modes. Some K giant stars can pulsate with much shorter periods. Hatzes \& Cochran (1994b) found evidence for pulsations with a period of 3.9 hours in $\beta$ Oph. Frandsen et al. (2002) detected possibly up to 9 modes in the period range $2-5.5$ hours with a mean separation of $7.2 \mu \mathrm{Hz}$. These were well matched by overtone radial modes. Recently, RV measurements of the giant $\epsilon$ Oph revealed excess power in the frequency spectrum corresponding to periods $\approx 4.6$ hours (de Ridder et al. 2006).

Oscillations in giant stars have also been established through photometric studies. Edmonds \& Gilliland (1996) used the Hubble Space Telescope to find variations in several $\mathrm{K}$ giants in 47 Tuc on time scales of a few days with semi-amplitudes of $10-30 \mathrm{mmag}$ in $U$. Buzasi et al. (2000) found 10 oscillation modes in $\alpha$ UMa (KO III) using the star camera of the Wide Field Infrared Explorer (WIRE) satellite. The modes had amplitudes of $100-$ $400 \mu \mathrm{mag}$ and the lowest frequency mode was at $1.82 \mu \mathrm{Hz}$ (6.36 days). Retter et al. (2003), also using the WIRE guide camera, found around four photometric modes in $\alpha$ Boo with periods ranging from $2-4$ days. However, they noted that these could be consistent with one mode with a short lifetime. 
Photometric variations on time scales of hours have also been found in $\mathrm{K}$ giant stars. Kallinger et al. (2005) found evidence for three approximately equidistant frequencies in the K2.5 giant star GSC 09137-03505 based on photometry made with the Fine Guidance Camera of HST. The frequency range for these were $21-71 \mu \mathrm{Hz}(0.163-0.55$ days $)$ with amplitudes of $291-341 \mathrm{ppm}$. However, these frequencies were significantly above the acoustic cutoff frequency casting some doubts as to their origin.

Early evidence points to the oscillations in $\mathrm{K}$ giants stars being caused by fundamental or overtone radial modes. The periods found in $\alpha$ Boo and $\beta$ Oph were consistent with fundamental or overtone radial modes (Hatzes \& Cochran 1994a, 1994b). The 10 modes found in $\alpha$ UMa by Buzasi et al. (2000) were also identified as radial modes (Guenther et al. 2000). The 0.57-day RV period in $\alpha$ Ari is also consistent with an overtone radial mode (Kim et al. 2006). However, ground-based observations over a short time span may be giving us an incomplete picture of oscillations in giant stars.

\section{New Results}

We conducted observations spanning 9 nights on a sample of fifteen $\mathrm{K}$ giant stars using the 2Dcoude spectrograph of the $2.7 \mathrm{~m}$ telescope of McDonald Observatory. Precise stellar radial velocity measurements were obtained using an iodine absorption cell placed in the light path of the telescope. The use of iodine cells has become a common technique for the measurement of precise stellar radial velocities (see Endl et al. 2000). The strategy of the observations was to look at a modest sample of $\mathrm{K}$ giant stars that spanned a wide range of spectral parameters rather than concentrating on a few objects. The sample size forced us to have a time sampling that may miss some modes. However, we were primarily interested in understanding how the characteristics of the stellar oscillations varied among $\mathrm{K}$ giant stars. Future observations would concentrate on extensive observations of a few interesting targets. Objects were chosen from the sample of Döllinger et al. (2007) which showed significant RV variations on short time scales. Our preliminary analysis showed that the oscillations in $\mathrm{K}$ giant stars could be divided into two classes: stars that show periods of several hours, and stars showing periods of several days. We present here two representative examples from this study.

\section{$\beta$ Oph}

Eighty-nine observations were made of $\beta$ Oph spanning nine days. Figure 1 shows the LombScargle periodogram of the RV measurements as well as the data window. There is significant power near frequencies of about $3.5 \mathrm{~cd}^{-1}(=40 \mu \mathrm{Hz})$. The highest peak at a frequency of $3.43 \mathrm{~cd}^{-1}(=39.7 \mu \mathrm{Hz})$ is statistically significant having a false alarm probability (chance that it is due to noise), FAP $=6.5 \times 10^{-5}$. This was assessed by randomly shuffling the $\mathrm{RV}$ measurements, keeping the times fixed and computing a periodogram over the frequency range shown in the figure. The number of random noise periodograms after a large number of shuffles $\left(2 \times 10^{5}\right)$ having power larger than the observed power yielded the FAP.

Additional frequencies were searched using a pre-whitening procedure. The program Gaussfit (Jefferys et al. 1988) was used to fit a sine function to the RV data. The frequency found by the periodogram analysis was used as an initial guess, but Gaussfit was allowed to vary the period, amplitude, and phase of the data to obtain a least-squares solution. A periodogram analysis was then performed on the residual RV data. A second significant peak was found at a frequency of $34.72 \mu \mathrm{Hz}$. The false alarm probability over the same frequency interval was again assessed using periodograms of randomly shuffled data sets. After $2 \times 10^{5}$ shuffles there was no instance where the random data periodogram had power greater than the residual data periodogram. This implies FAP $<5 \times 10^{-6}$. An additional application of this procedure by subtracting the contribution of the $34.72 \mu \mathrm{Hz}$ frequency yielded a highest 


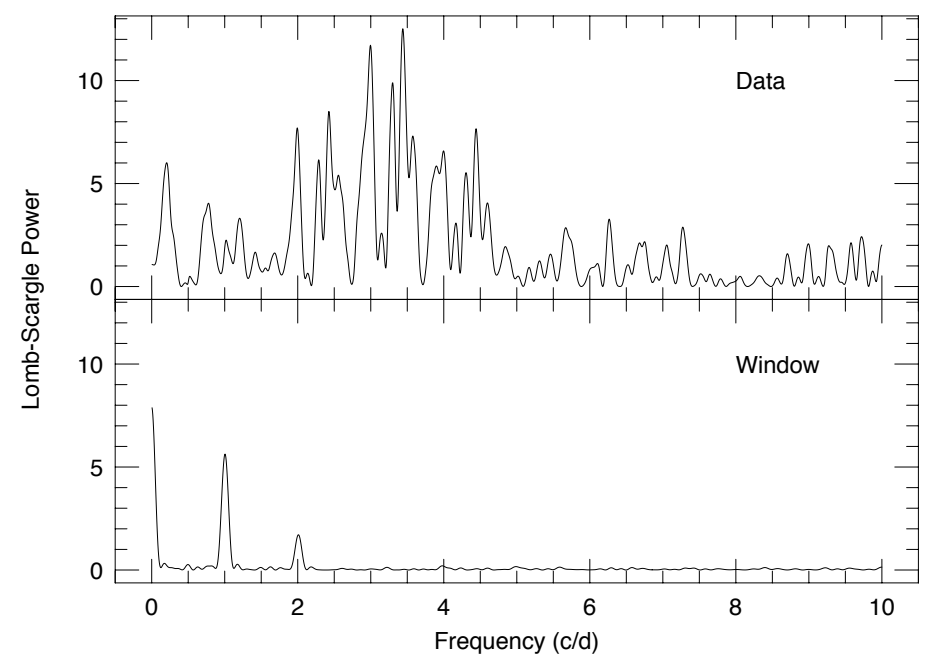

Figure 1: The Lomb-Scargle periodogram of the RV measurements of $\beta$ Oph. The highest peak has a false alarm probability of $6.5 \times 10^{-5}$.

\begin{tabular}{ccc}
\hline $\begin{array}{c}\text { Frequency } \\
(\mu \mathrm{Hz})\end{array}$ & $\begin{array}{c}\text { Period } \\
(\text { hrs })\end{array}$ & $\begin{array}{c}\text { Amplitude } \\
\mathrm{m} \mathrm{s}^{-1}\end{array}$ \\
\hline 38.79 & 6.98 & 7.43 \\
34.72 & 8.00 & 7.39 \\
\hline
\end{tabular}

Table 1: Detected pulsation modes in $\beta$ Oph

peak at $40.97 \mu \mathrm{Hz}$, but this had low significance (FAP $=0.03$ assessed with random data periodograms). Table 1 lists the two most significant detected frequencies.

$\gamma$ Dra

Eighteen RV measurements were made of $\gamma$ Dra over nine nights. The top panel of Fig. 2 shows the time series of these measurements. It is clear that $\gamma$ Dra shows stellar oscillations with much longer periods and larger amplitudes than $\beta$ Oph. There are clear deviations of the RV measurements from the dominant period of 3.97 days.

A prewhitening procedure was also performed on the $\gamma$ Dra data by finding the leastsquares sine-fit to the data using Gaussfit, subtracting this component, and performing a sine-fit on the residuals. This is shown in the lower panels of Fig. 2. After finding 3 sine components the final residual in the RV values was $\sigma=5.8 \mathrm{~m} \mathrm{~s}^{-1}$ which is comparable to the mean error of the measurements (mean $\sigma=5.7$ ). The pulsation modes found in $\gamma$ Dra are listed in Table 2. For one set of residuals (third panel from top in Fig. 2) two periods could fit the data: 2.5 days, or an alias of 1.6 days (shown as a dashed line in the figure). The 2.5-day period provided a slightly better fit although the sampling of our data is such that we do not know if the 2.5 or the 1.6-day period is actually present in the data.

The false alarm probabilities of the detected peaks in $\gamma$ Dra were determined using the "random shuffle" technique described above and $2 \times 10^{5}$ shuffles. This resulted in FAP $=$ $0.0004,0.034$, and 0.0072 for the $4.02,9.09$, and 2.5 -day periods, respectively. We should mention several caveats. After subtraction of the dominant 4-day period there are clear longterm variations in the RV residuals, but with a period that is comparable to the time span 


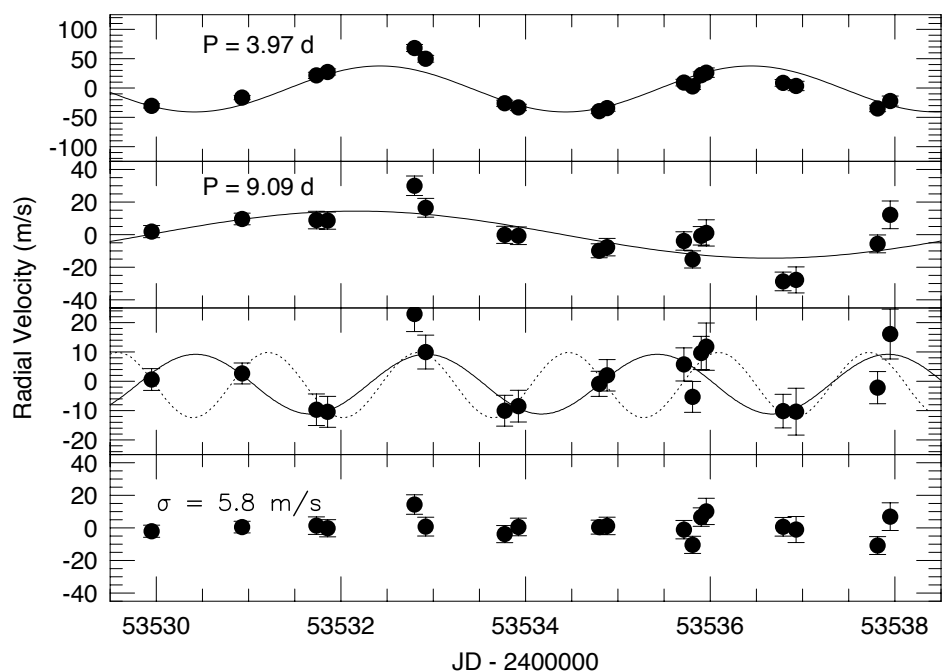

Figure 2: RV measurements of $\gamma$ Dra taken on 9 consecutive nights. Each panel shows successive steps of the pre-whitening procedure. In the second panel from the bottom the best fit period of 2.5 days is shown as a solid line. The dashed line shows an alias period of 1.62 days.

\begin{tabular}{ccc}
\hline $\begin{array}{c}\text { Frequency } \\
(\mu \mathrm{Hz})\end{array}$ & $\begin{array}{c}\text { Period } \\
\text { (days) }\end{array}$ & $\begin{array}{c}\text { Amplitude } \\
\mathrm{m} \mathrm{s}^{-1}\end{array}$ \\
\hline 2.89 & 4.02 & 39.7 \\
1.27 & 9.09 & 14.4 \\
4.63 & 2.50 & 10.2 \\
\hline
\end{tabular}

Table 2: Detected pulsation modes in $\gamma$ Dra

of the data. This period is uncertain. This is also probably reflected in the rather large FAP since Lomb-Scargle periodograms tend to give rather high FAPs for partial sine waves, even though a real signal is in the data. Although the FAP for the 2.5-day period is rather low our data sampling is sparse so this may be uncertain as well.

Table 3 lists the basic stellar parameters for $\beta$ Oph and $\gamma$ Dra. Temperatures are from McWilliam (1990) and the masses and radii were determined using the method outlined in da Silva et al. (2006). Also listed is the period of the fundamental radial mode for each star, derived from the empirical relationships of Cox, King \& Stellingwerf (1972).

\begin{tabular}{lll}
\hline Parameter & $\gamma$ Dra & $\beta$ Oph \\
\hline Radius $\left[R_{\odot}\right]$ & 47 & 14 \\
Mass $\left[M_{\odot}\right]$ & 2.9 & 2.6 \\
$\mathrm{~T}_{\text {eff }}[\mathrm{K}]$ & 4550 & 3903 \\
$F($ days $)$ & 8.4 & 1.2 \\
Amplitude $\left(\mathrm{m} \mathrm{s}^{-1}\right)$ & 41 & 5.6 \\
$\nu_{0}(\mu \mathrm{Hz})$ & 0.71 & 4.2 \\
\hline
\end{tabular}

Table 3: Stellar Parameters for $\gamma$ Dra and $\beta$ Oph. $\mathrm{F}$ is the radial fundamental mode period and $\nu_{0}$ is the asymptotic p-mode frequency spacing 


\section{Discussion}

Kjeldsen \& Bedding (1995) proposed a scaling relationship for the the velocity amplitudes, $V$, for stellar oscillations that was proportional to the ratio of stellar luminosity to mass $(V \propto$ $L / M) . \beta$ Oph and $\gamma$ Dra have comparable masses. $\gamma$ Dra has a higher effective temperature and has approximately 10 times the surface area. Its luminosity should thus be $10-20$ times greater than that of $\beta$ Oph. The amplitudes for the oscillations seen in $\gamma$ Dra are a factor of ten higher than for $\beta$ Oph which is more or less consistent with the Kjeldsen \& Bedding relation. Table 3 also lists the predicted RV amplitudes for $\beta$ Oph and $\gamma$ Dra using the Kjeldsen \& Bedding scaling law. Both the time scales and amplitudes are consistent with solar-like p-mode oscillations given the radius and mass for each star.

For p-mode solar-like oscillations the frequency spacing scaled to solar parameters is $\nu_{0}=$ $135 M_{*}^{1 / 2} R_{*}^{-3 / 2} \mu \mathrm{Hz}$, where the mass and radius $(M$ and $R$ ) are in solar units (Brown \& Gilliland 1994). For high order modes $(n>>1)$ p-modes are evenly spaced with a frequency spacing of $\nu_{0} / 2$. Table 3 also lists the values $\nu_{0}$.

If we are to believe the additional periods found in $\beta$ Oph and $\gamma$ Dra, then we can use these for a rough estimate of the frequency spacing. This "spacing" is $1.7 \mu \mathrm{Hz}$ for $\gamma$ Dra and $4.3 \mu \mathrm{Hz}$ for $\beta$ Oph, both within a factor of 2-3 from the expected spacing. However we should be cautious in interpreting the separation of the modes to $\nu_{0} / 2$. More modes need to be detected to get a better measurement of the frequency spacing.

The periods we have detected are comparable to those of the fundamental radial modes for these stars suggesting that these may be radial as opposed to nonradial modes. The classical definition of the pulsation constant is $Q=P\left(M / M_{\odot}\right)^{0.5}\left(R / R_{\odot}\right)^{-1.5}$ where $\mathrm{M}$, and $\mathrm{R}$ are the stellar mass and radius, respectively. This results in pulsation constants of $Q=0.048,0.0212$, and 0.0132 for the 3 modes of $\gamma$ Dra (in increasing frequency) and $Q=0.013$ and 0.0114 for the two highest amplitude (and thus most significant) modes in $\beta$ Oph. Using the theoretical models of Guenther et al. (2000) the 3 modes in $\gamma$ Dra can be identified with the fundamental, first, and third overtone modes, respectively $(n=0,1$, and 4$)$. The two modes in $\beta$ Oph are consistent with the 4 and 5 th overtone mode $(n=4$ and 5). This is consistent with radial modes inferred for other $\mathrm{K}$ giant stars.

In spite of over a decade of (admittedly intermittent) studies of variability of giant stars we still know little about oscillations in giant stars. We do know that giant stars vary with periods ranging from hours to several days, but the exact mode identification remains elusive. There are hints of mode switching but the lifetimes of the modes are unknown. Possibly the one thing we can be sure of about these stars is that we probably have yet to derive the full oscillation spectrum for even a single giant star. The reason is clear, with pulsation periods ranging up to several days it is difficult to derive such a spectrum without extensive time coverage using multi-site campaigns.

This will change with the launch of the CoRoT satellite. This French-led mission (with participation from Austria, Belgium, Brazil, Germany, Spain, and ESA) has the dual goal of asteroseismology and the detection of transiting planets. Ultra-precise light curves will be obtained for thousands of stars and among them many $\mathrm{G}-\mathrm{K}$ giants. Observations will be made continuously for up to 150 days. CoRoT will, for the first time, be able to derive the full oscillation spectrum for many giant stars as well as to determine the mode lifetimes.

Acknowledgments. This work is based on observations made with the $2.7 \mathrm{~m}$ Harlan J. Smith Telescope at McDonald Observatory. The authors would like to thank the anonymous referee for suggested improvements to the manuscript. The observations at McDonald Observatory were made possible through grant HA 3279/4-1 of the Deutsche Forschungsgemeinschaft (DFG). 


\section{References}

Brown T. M., Gilliland R. L., 1994, ARA\&A, 32, 37

Buzasi D., Catanzarite J., Laher R., et al., 2000, ApJ, 532, L133

Cox J. P., King D. S., Stellingwerf R. F., 1972, ApJ, 171, 39

da Silva L., Girardi L., Pasquini L., et al., 2006, A\&A, 458, 609

de Ridder J., Barban C., Carrier F., et al., 2006, A\&A, 448, 689

Döllinger, M. P., Hatzes A. P., Pasquini L., et al., 2007, A\&A, in press

Edmonds P. D., Gilliland R. L., 1996, ApJ, 464, L157

Endl M., Kürster M., Els S., 2000, A\&A, 362, 585

Frandsen S., Carrier F., Aerts C., et al., 2002, A\&A, 394, L5

Frink S., Mitchell D. S., Quirrenbach A., et al., 2002, ApJ, 576, 478

Guenther D. B., Demarque P., Buzasi D., et al., 2000, ApJ, 530, L45

Hatzes A. P., Cochran W. D., 1993, ApJ, 413, 339

Hatzes A. P., Cochran W. D., 1994a, ApJ, 422, 366

Hatzes A. P., Cochran W. D., 1994b, ApJ, 432, 763

Hatzes A. P., Guenther E. W., Endl M., et al., 2005, A\&A, 437, 743

Hatzes A. P., Cochran W. D., Endl M., et al., 2006, A\&A, 457, 335

Jefferys W. H., Fitzpatrick M. J., McArthur B. E., 1988, Celest. Mech., 41, 39

Kallinger Th., Zwintz K., Pamyatnykh A. A., Guenther D. B., Weiss W. W., 2005, A\&A, 433, 267

Kim K. M., Mkrtichian D. E., Lee B.-C., Han I., Hatzes A. P., A\&A, 2006, 454, 839

Kjeldsen H., Bedding T. 1995, A\&A, 293, 87

McWilliam A., 1990, ApJS, 74, 1075

Retter A., Bedding T. R., Buzasi D. L., Kjeldsen H., Kiss L. L., 2003, ApJ, 591, L151

Setiawan J., Hatzes A. P., von der Lühe O., et al., 2003, A\&A, 398, L19

Setiawan J., Rodmann J., da Silva L., et al., 2005, A\&A, 437, L31

Smith P. H., McMillan R. S., Merline W. J., 1987, ApJ, 317, L79

Walker G. A. H., Yang S., Campbell B., Irwin A. W., 1989, ApJ, 343, L21

\section{DISCUSSION}

Matthews: Considering the giants that we already observed with MOST, supported by some ground-based spectroscopic observations, and that we will be observing NGC 752 with $\sim 20$ giants in different evolutionary stages, we share your feeling that every giant will show variability and possibly every giant will show p-mode oscillations. 


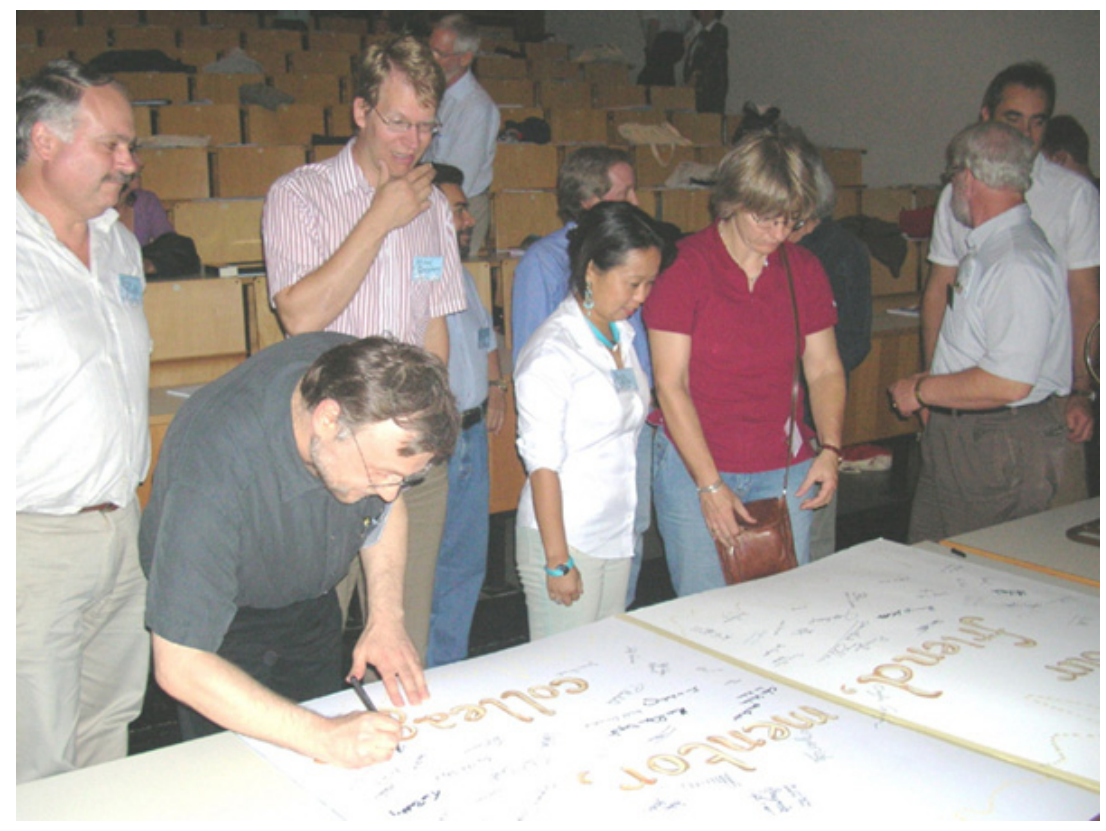

Ennio Poretti, Günter Houdek, Atsuko Nitta and Judi Provencal signing Michel Breger's birthday card; Mike Montgomery still wonders where to sign.

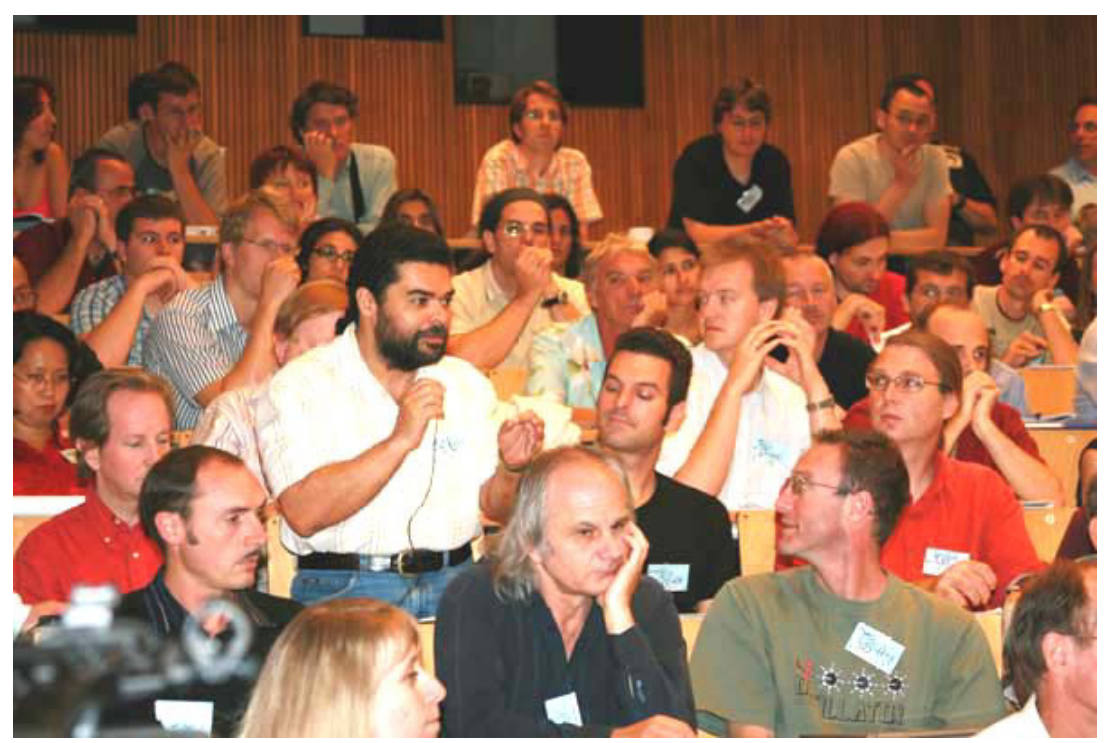

As usual, Kepler makes his point very clear. 